\title{
Rett syndrome: a seminal book on extensive multidisciplinary analyses for rare disease
}

\author{
Pierre Bitoun ${ }^{1}$ \\ Received: 24 March 2018 / Accepted: 20 April 2018 / Published online: 25 May 2018 \\ (c) European Society of Human Genetics 2018
}

Rett Syndrome

Edited by: Walter E Kaufman

Published by: MacKeith Press Ltd.

Year: 2017

ISBN: 9781909962835

Price: $£ 70.00$

This book is unique in its overall broad spectrum describing RETT syndrome (RTT) from the history of its identification to the detailed clinical, communication, cognitive and behavioral features, their temporal evolution, its medical and surgical management, molecular genetics, research findings leading to therapeutic trials, and including a parental experience description.

As such, this precious compendium skillfully crafted by 40 expert authors led by five editors can be thought of as a model for the comprehensive approach needed for rare disease syndrome management by professionals in pediatrics, neurosciences, genetics, physiology, physiotherapy, rehabilitation, and drug discovery.

After reviewing the vast contribution of Andreas Rett in the identification of a neurodevelopmental disorder affecting girls with a halt in the development and hand stereotypies, initially mistakenly thought to be linked to hyperammonemia in 1966 and 1977, followed by the seminal paper of Bengt Hagberg, Jean Aicardi et al. describing a large series of 35 girls with loss of purposeful hand movements in 1983 leading to the successive consensus diagnostic criteria of 1998, 2001a (Kerr et al.), and finally 2010 by Jeffrey Neul et al. These clinical expert syndrome delineations were comforted by the identification of the MECP2 causal gene by Amir et al in 1999, followed

Pierre Bitoun

bitoun@gmail.com

1 Groupe Medical Jarente, Paris, France by the identification of the variant RTT-associated genes, CDKL5 and FOXG1.

The current diagnostic description emphasizes the dynamic temporal evolution of RTT, with five phases of normal development for 6-18 months, development arrest, regression of social contact and finger skills, stabilization with better social contact, and eye gaze by age 5 in most, and thereafter effective social interaction with gradual slowing of motor functions through adulthood.

The consensus diagnostic criteria, which should be considered in girls with a period of regression, followed by recovery or stabilization include two of the four main criteria and five of the 11 supportive criteria, and exclusion of traumatic brain injury and grossly abnormal development before 6 months (lack of head control, swallowing or social smile). The main criteria includes loss of purposeful hand skills or acquired spoken language, gait abnormalities, and stereotypic hand movements. Supportive criteria include awake breathing anomaly or bruxism, sleep pattern, muscle tone, peripheral vasomotor, kyphoscoliosis, short stature, small cold hands and feet, laughing/screaming spells, and intense eye communication.

The book clearly describes the difference between "classical RTT"associated with MECP2 mutations in over 90\% versus the variant forms of "preserved speech" also mostly linked to MECP2, while the "early seizure" variant should prompt genotyping of CDLK5, and the congenital variant is mostly associated with FOXG1 mutations.

The evaluation of cognition, communication, and behavior skills are described in detail, with a particular discussion of adequate tools for assessment in persons with altered speech and development.

The structure of MECP2 is discussed in detail with methylated-CpG-binding domain harboring $65 \%$ of pathogenic amino acid substitutions, while eight $\mathrm{C}$ to $\mathrm{T}$ transitions at hotspots account for $70 \%$ of the mutations found in RTT, 7-12\% are small insertions/deletions found in the C-terminal domain, and $7 \%$ are large deletions. Use of mutation-type to assess the severity is discouraged, even 
if certain variants are associated with risk of scoliosis or seizures, because of the multiple modifiers known and unknown affecting transcription. More than 300 MECP2 mutations have been described.

The particular properties of the MECP2 protein are clearly explained with its role in epigenetic control, as well as its disordered protein nature enabled by a particular amino acid sequence allowing flexible three-dimensional (3D) conformation for adaptation to multiple DNA target sequences and interaction with multiple cofactors. MECP2 only binds to $2 \%$ of highly methylated gene promoters, its role may be more to modulate active gene expression by binding away from the promoters (60\% of binding sites), rather than silencing the inactive genes. MECP2 enhances the formation of large chromatin loops, thereby regulating large groups of genes, particularly in neurons and glial cells to control precise programs of transcription and repression in response to neuronal activity. There are nearly as many MECP2 molecules as nucleosomes to regulate global chromatin structure.

The mechanism of variation of clinical presentation of RTT is exquisitely described with $\mathrm{X}$ chromosome inactivation, which can be reliably assessed from peripheral lymphocytes, as proven to be concordant with brain inactivation, the four 3'UTR variant isoforms could be a major modifier including mutations in this region, which could cause RETT as well as other neurodevelopmental disorders, as evidenced by the variants found in the $3^{\prime} \mathrm{UTR}$ in subjects with autism spectrum disorders. Mir 132 found to bind to $3^{\prime} \mathrm{UTR}$ regulating MECP2 expression Trans modifiers such as BDNF and CNTNAP2 are prominent as well as multiple other gene variants.

MECP2 is a regulator of regulators interacting with BDNF,CREB1,ID1,2,3,4, RET, EGR2, MEF2C, and MiR137 controlling epigenetics of neurodevelopmental pathways in health and disease.

Orthopedic issues and their management are described in detail including scoliosis, hip dysplasia-which may occur in up to $37 \%$ of girls and women-foot deformities and the possible role of decreased muscle tone. The prevention with back muscle extensors physiotherapy and surgical management of scoliosis is presented in detail with case studies showing the impact of hospitalization on development and discussion of choices between Posterior Segment Instrumentation and Fusion (PSIF) versus more recent evolutive techniques of Vertical Expandable Prosthetic Titanium Rib. Detailed advice as to what is known and what needs further research to improve orthopedic management roadmap is presented.

Sleep issues that are part of neuroautonomic dysfunction, including breathing disturbances, are also described at length and include difficulty in going to sleep, prolonged daytime sleep, night awakenings, and increased sleep time.
This disturbance seems due to dopaminergic hypofunction, followed by receptor supersensitivity in the striatum. The putative arrested brain development could cause the infantile prolonged sleep pattern, daytime sleep, and circadian rhythm disturbance. Case studies suggest the need for careful sleep evaluation and pharmacologic management with melatonin or others, since they involve over $70 \%$ of subjects.

Epilepsy is discussed broadly as the major morbidity of RTT and involves 60-90\% of subjects and onset before age 5 predicting intractability and severity. Infantile spasm or hypsarythmia is a hallmark of the CDKL5 mutations. Specific central spikes, synchronous to hand tapping movements, are considered non epileptic, while centrotemporal spikes accentuating in sleep with slow background are pathognomonic. Specific mutations in the methyl binding domain or nuclear localization signal are associated with earlier and severe epilepsy. The val/val BDNF variant is associated with milder epileptic course. The drug of choice seems to be valproate or lamotrigine, but topiramate and carbamazepine (in older subjects) seems to be the adequate second line drugs.

Breathing disorders are specific to RTS and include breathing irregularity, hyperventilation with decreased inspiratory and expiratory duration, as well as specific breath holding in inspiration with tachycardia, as opposed to the usual bradycardia that reflect central dysautonomia.

Results of MeCP2- mouse model experiments are described showing enhancement of GABA-A receptor, serotoninergic HT1a receptor, and BDNF signaling all improving breathing disturbances. The role of MeCP2 in the maintenance of respiratory circuitry in adults as well as that of the glutamatergic Kolliker Fuse nucleus in lung inflation / glottal closure coordination control are discussed in detail. Whether the progression of respiratory dysfunction causes the disease progression or is simply a reflection of loss of neuronal control is still unclear. It however results in oxidative and hypoxic stress.

Growth, nutrition, and bone health are discussed at length, since head circumference, height, and weight for age deviate below the reference population, respectively, at 1 , 17 , and 6 months, but not BMI, and there is no pubertal growth spurt, possibly reflecting the lack of mandatory metabolic energy increase. Prevalence of growth failure is unknown, but is reported in $45 \%$ of the affected individuals. Bone age can be advanced or delayed, but all standard hormonal testing seems to be normal including $\mathrm{GH}$ and fasting cortisol, but afternoon cortisol, urinary cortisol, leptin, and adiponectin are increased, while Ghrelin levels are low in females with RTT. Weight issues are linked to lack of dietary energy intake due to behavior, oral motor dysfunction, involuntary movements, bruxism, breathing problems, and inability to self feed. Supplemental oral 
feeds, increased energy intake, and as needed gastrostomy "buttons" can be used. Dietary calcium and vitamin D intakes must be ascertained closely to insure prevention of fractures common in subjects with RTT.

One can only regret the book does not include a gene structure diagram nor a list of web-based RTT resources for clinicians, researchers, and families.

Finally a detailed list of treatments being tested in clinical trials is given with their physiologic rational including HDAC6 inhibitors, which restore microtubule network stability, statins, or bone marrow transplant, as well as a large body of studies on animal models paving the way to therapy.
The authors describe a paradigm shift now defining RTT as a disorder of neuronal plasticity, and the possibility of restoration makes RTT a potentially treatable disorder.

This book constitutes a prototype for rare disease syndrome description with its large collaborative authorship, its broad spectrum of specialties, its inclusion of parental descriptions and case studies, its very thorough clinical and scientific analysis of all features of the disorder, its therapeutic outlook, and its practical advices for all healthcare workers. 